\title{
Zeitmanagement und Lebensqualität nach Umstellung einer topischen und/oder Fototherapie auf Fumar- säureester bei Patienten mit Psoriasis
}

\author{
Time Management and Quality of Life After Change of Therapy from Topical \\ Treatment and/or Phototherapy to Fumaderm ${ }^{\circledR}$ in Patients with Psoriasis
}

Autoren

Institut
C. Kahl, M. Neureither, K. Reich, I. Zschocke

SClderm GmbH, Hamburg
Bibliografie

DOI http://dx.doi.org/

$10.1055 / \mathrm{s}-0030-1256613$

Akt Dermatol 2011; 37:

249-253 @ Georg Thieme

Verlag KG Stuttgart · New York

ISSN 0340-2541

Korrespondenzadresse

Dr. med. Christina Kahl

Fachärztin für Dermatologie und Venerologie

SCIderm GmbH

Esplanade 6

20354 Hamburg

christina.kahl@sciderm.com

\section{Zusammenfassung \\ $\nabla$}

Einführung: Die Psoriasis geht häufig mit einer Einschränkung der Lebensqualität betroffener Patienten einher. Die Einschränkung der Lebensqualität scheint dabei am engsten mit dem Zeitaufwand zu korrelieren, den ein Patient für die Behandlung der Psoriasis aufwenden muss. Mit Hilfe dieser Studie sollte daher untersucht werden, ob eine Therapieumstellung von topischer und/oder Fototherapie auf Fumaderm ${ }^{\circledR}$ mit begleitender optionaler Lokaltherapie zu einer zeitlichen Reduktion des Behandlungsaufwandes und zu einer Besserung der Lebensqualität und des Schweregrades der Psoriasis bei betroffenen Patienten führt.

Material und Methode: Im Rahmen dieser nichtinterventionellen Studie wurden Patienten mit einer moderaten bis schweren Psoriasis beobachtet, die von einer topischen und/oder Fototherapie auf eine Behandlung mit Fumaderm ${ }^{\circledR}$ mit begleitender optionaler Lokaltherapie umgestellt wurden. Vor und nach ca. 12-wöchiger Therapie mit Fumaderm ${ }^{\circledR}$ wurden der PGA, der DLQI sowie die Dauer der täglichen Psoriasis-Behandlung be-

\section{Einführung}

\section{$\nabla$}

Mit einer Prävalenz von 2,1-2,5\% und ca. 2 Millionen betroffenen Patienten stellt die Psoriasis eine der häufigsten chronisch-entzündlichen Erkrankungen in Deutschland dar [1].

Bei Patienten mit ausgedehnteren Hautveränderungen geht die Erkrankung mit einer deutlichen Einschränkung der Lebensqualität einher $[2,3]$. Sie ist dabei der Lebensqualitätsminderung bei Patienten mit chronischen Erkrankungen wie Bluthochdruck oder Diabetes vergleichbar [2]. Nicht selten zeigt sich außerdem eine Assoziation mit psychiatrischen Problemen. So leidet nahezu stimmt. Zudem wurde erfasst, in welchem Ausmaß acht Aussagen zur Behandlungsbelastung unter besonderer Berücksichtigung des Zeitaufwandes - zutreffen.

Ergebnisse: Nach ca. 12-wöchiger Therapie mit Fumaderm ${ }^{\circledR}$ konnte bei den 24 eingeschlossenen Patienten eine durchschnittliche zeitliche Reduktion der täglichen Behandlungsdauer festgestellt werden. Auch sieben der acht Aussagen zur Behandlungsbelastung deuteten auf eine Besserung der zeitlichen Belastung hin. Durchschnittlich sank der PGA von 3,2 auf 1,75, der DLQI-Gesamtwert von 11,2 auf 4,4. Insgesamt wurden elf zumindest möglicherweise mit der Studienmedikation assoziierte unerwünschte Ereignisse dokumentiert.

Diskussion: Die Ergebnisse weisen darauf hin, dass eine Therapie mit Fumaderm ${ }^{\circledR}$ mit begleitender optionaler Lokaltherapie weniger zeitaufwendig ist als eine topische und/oder Fototherapie. Zudem konnte eine deutliche Verbesserung der Lebensqualität und der Schwere der Schuppenflechte-Erkrankung nach 12-wöchiger Behandlung mit Fumaderm ${ }^{\circledR}$ festgestellt werden.

die Hälfte der Psoriasis-Patienten an Depressionen oder Ängsten [4].

Um die Lebensqualität bei Patienten mit Hauterkrankungen zu beurteilen, wurde 1994 ein spezieller Fragebogen, der „Dermatology Life Quality Index“ (DLQI), entwickelt und im Rahmen klinischer Studien validiert [5]. Der DLQI besteht aus 10 Fragen, die die Symptome und Therapie der Hauterkrankung sowie deren Auswirkung auf die täglichen Aktivitäten des Patienten, das Freizeitverhalten, den Berufsalltag und die sozialen Beziehungen berücksichtigen. Der Fragebogen wird mittlerweile routinemäßig neben Parametern, die die Schwere der Hautbeteiligung beschreiben (z. B. 


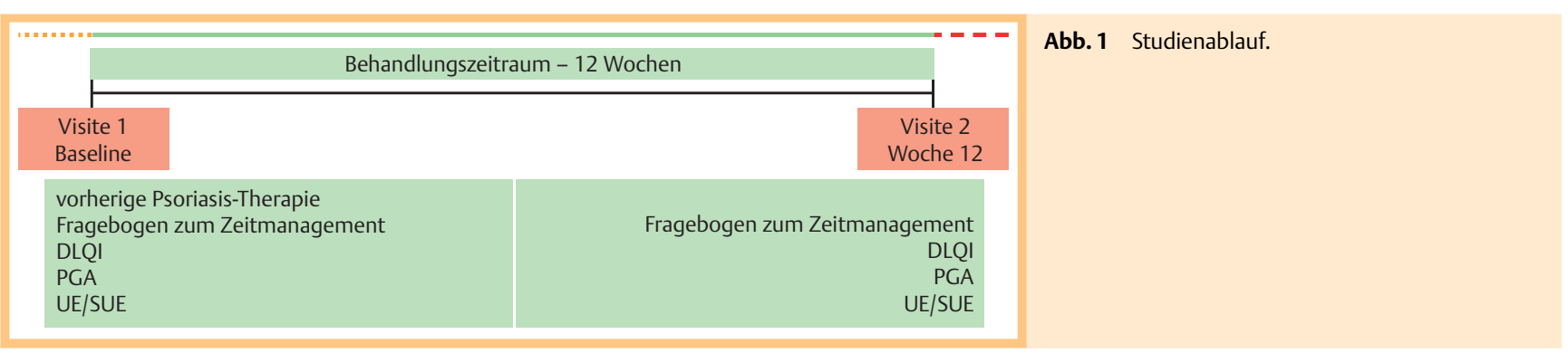

Psoriasis Area and Severity Index [PASI], Physician's Global Assessment [PGA], Body Surface Area [BSA]), in klinischen Studien zur Messung des Therapieerfolges bei Psoriasis-Patienten eingesetzt. In einer Arbeit von Blome et al. aus dem Jahre 2010 [6], in der die Korrelation von 12 Prädikatoren mit der Lebensqualität von Psoriasis-Patienten untersucht wurde, konnte gezeigt werden, dass die Zeit, die ein Patient täglich für die Behandlung seiner Schuppenflechte aufwenden muss, am stärksten mit der Lebensqualität korreliert. Erst danach folgten der patientendefinierte Behandlungsnutzen, die Schwere der Hauterkrankung sowie die Zufriedenheit mit der Behandlung.

Um eine Besserung der Lebensqualität bei Psoriasis-Patienten zu erzielen, sollte bei der Therapieauswahl daher neben der eigentlichen Effektivität immer berücksichtigt werden, wie praktikabel eine Behandlung - vor allem im Bezug auf den Zeitaufwand - für den Patienten ist.

So wurde im Rahmen der nachfolgend beschriebenen Studie unter besonderer Berücksichtigung der Zeitersparnis - der Nutzen einer Therapieumstellung von topischer und/oder UVBehandlung auf Fumaderm ${ }^{\circledR}$ bei Patienten mit Schuppenflechte untersucht.

\section{Material und Methoden}

$\nabla$

Die prospektive, multizentrische, nicht-interventionelle Studie (NIS) wurde im Zeitraum von Dezember 2009 bis April 2010 durchgeführt und umfasste insgesamt zwei Visiten pro Proband. Beobachtet wurden erwachsene Patienten mit einer moderaten bis schweren Schuppenflechte (PGA 3 oder 4) und einer mindestens zwei Wochen durchgeführten topischen und/oder Fototherapie, die auf eine systemische Therapie mit Fumaderm ${ }^{\circledR}$ umgestellt wurden. Die topische Therapie durfte dabei auf Wunsch des Patienten fortgesetzt, die Fototherapie musste beendet werden. Die erste Visite im Rahmen der Studie wurde am Tag der Therapieumstellung durchgeführt. Die zweite und letzte Visite sollte gemäß dem Prüfprotokoll 12 Wochen später stattfinden. Bei beiden Visiten wurde zur Festlegung des Schweregrades der Psoriasis der PGA durch den behandelnden Arzt bestimmt und zur Einschätzung der Lebensqualität der DLQI-Fragebogen vom Probanden ausgefüllt $(\bullet$ Abb. 1).

Die Probanden sollten außerdem dokumentieren, wie viel Zeit sie für die topische und/oder Fototherapie (Visite 1) bzw. für die Systemtherapie mit Fumaderm ${ }^{\circledR}$ mit oder ohne Kombination einer Lokaltherapie (Visite 2) ihrer Erkrankung täglich benötigen. Zudem wurden sie gebeten einzuschätzen, in welchem Ausmaß („trifft nicht zu“, „trifft eher nicht zu“, ,trifft eher zu“, ,trifft vollständig zu“) die folgenden acht Aussagen zur Belastung durch die Psoriasis-Behandlung unter besonderer Berücksichtigung des Zeitaufwandes bei ihnen zutreffen:
1. „Aufgrund der Psoriasis-Behandlung muss ich morgens früher aufstehen.“

2. „Die jetzige Behandlung hat mir geholfen, weniger auf Arztbesuche angewiesen zu sein.“

3. „Für die Durchführung meiner Behandlung benötige ich fremde Hilfe."

4. „Die Behandlung beeinflusst mich in meiner Kleiderwahl.“

5. „Ich muss meine Kleidung aufgrund der Behandlung öfter als normal wechseln oder waschen."

6. „Im Rahmen meiner Behandlung habe ich oft Termine bei meinem Hautarzt.“

7. „Der Zeitaufwand für die Behandlung beeinträchtigt mein Leben.“

8. „Durch die Behandlung fühle ich mich in der Pflege sozialer Kontakte stark eingeschränkt.“

Unerwünschte Ereignisse (UE), schwerwiegende unerwünschte Ereignisse (SUE) sowie Begleittherapien wurden an beiden Visiten dokumentiert.

Bei Probanden, die die Studie vorzeitig beenden wollten, konnte die zweite Visite vorgezogen und eine „Early Termination“-Visite durchgeführt werden.

Hauptziel der Studie war es festzustellen, ob eine Therapie mit Fumaderm $^{\circledR}$ weniger zeitaufwendig ist als die vorausgegangene Behandlung mit topischer und/oder Fototherapie.

Nebenziele waren, die Effektivität von und die Veränderung der Lebensqualität durch Fumaderm ${ }^{\circledR}$ bei Patienten mit moderater bis sehr schwerer Psoriasis vulgaris im Vergleich zu der vorausgegangenen topischen und/oder Fototherapie zu beurteilen. $\mathrm{Zu}$ diesem Zweck wurden die DLQI- und PGA-Werte sowie die Einschätzung der Aussagen zur Belastung durch die Behandlung von Visite 1 und 2 verglichen. Die Auswertung der Daten erfolgte rein deskriptiv.

\section{Ergebnisse}

24 Probanden mit moderater bis schwerer Psoriasis wurden in die Studie eingeschlossen, darunter drei mit einer vorausgehenden Fototherapie (mit oder ohne zusätzlicher topischer Therapie).

Drei Probanden brachen die Studie nach 30 bis 40 Tagen ab; zwei aufgrund von Nebenwirkungen, einer aus persönlichen Gründen. Bei allen dreien wurde eine „Early Termination“-Visite durchgeführt und die Ergebnisse wurden in die Auswertung aufgenommen. Ein weiterer Proband, der nach Randomisierung keine Studienmedikation eingenommen hatte, wurde als „drop out“ behandelt. Die zu Visite 1 erhobenen Daten wurden in die Auswertung aufgenommen ( $\bullet$ Abb. 2).

Das durchschnittliche Alter der Probanden lag bei 47,8 Jahren, der durchschnittliche Abstand zwischen den beiden Visiten betrug 78,5 Tage ( $\bullet$ Tab. 1$)$. 


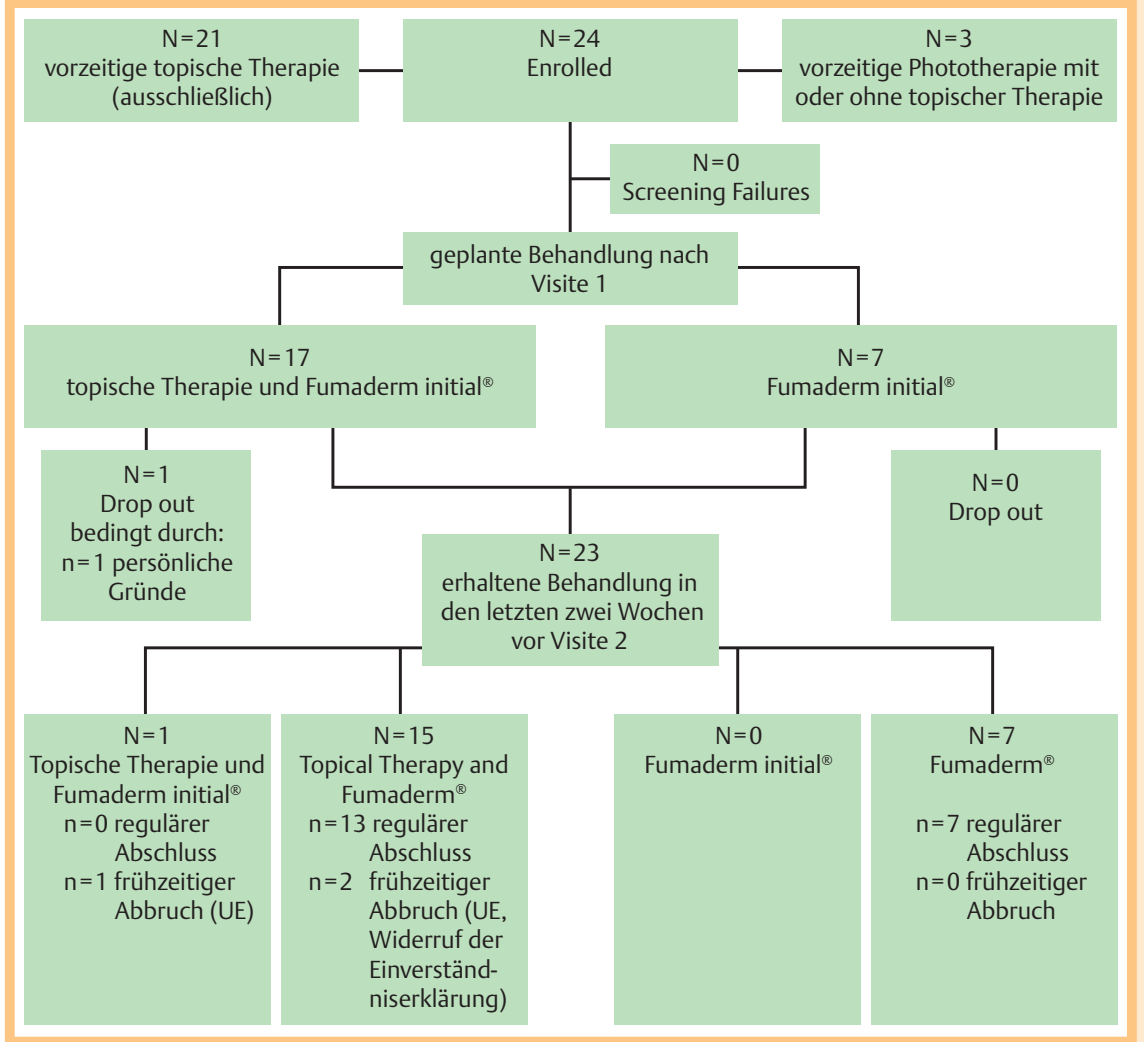

Abb. 2 Verteilung der Probanden.

Tab. 1 Demografische Daten der Probanden.

\begin{tabular}{|l|lc|}
\hline Geschlecht & Männlich & $15(63 \%)$ \\
\hline Alter in Jahren & Weiblich & $9(38 \%)$ \\
\hline & $n$ & 24 \\
\hline & Mean & 47.8 \\
\hline & SD & 14.89 \\
\hline & Median & 46.5 \\
\hline & Min & 25.0 \\
\hline Abstand zwischen Visite 1 und 2 in Tagen & Max & 73.0 \\
\hline & $n$ & 24 \\
\hline & Mean & 78.5 \\
\hline & SD & 25.53 \\
\hline & Median & 84.5 \\
\hline & Min & 1.0 \\
\hline & Max & 107.0 \\
\hline
\end{tabular}

Zu Visite 1 gaben 17\% der Probanden $(n=4)$ an, mehr als 45 Minuten pro Tag zur Behandlung ihrer Schuppenflechte zu benötigen, zu Visite 2 benötigte kein Proband länger als 45 Minuten pro Tag. Ebenso stieg die Anzahl der Probanden, die 30 oder weniger Minuten pro Tag für die Behandlung aufwandten, von $67 \%$ $(\mathrm{n}=16)$ auf $84 \%(\mathrm{n}=20)(\bullet$ Abb. 3$)$.

Bei insgesamt elf Probanden verringerte sich der Zeitaufwand zwischen Visite 1 und 2, bei acht Probanden zeigte sich keine Veränderung. Nur drei Probanden gaben an, mehr Zeit für die Fumaderm ${ }^{\circledR}$-Therapie mit begleitender optionaler Lokaltherapie als für die vorausgehende topische und/oder Fototherapie zu benötigen.

Auch konnte eine Verbesserung von sieben der acht Aussagen zur Belastung durch die Psoriasis-Behandlung unter besonderer Berücksichtigung des Zeitaufwandes von Visite 1 zu Visite 2 beobachtet werden: Der Anteil der Patienten, die die Aussagen als nicht zutreffend oder eher nicht zutreffend einstuften, stieg von
Visite 1 zu Visite 2 bei „Aufgrund der Psoriasis-Behandlung muss ich morgens früher aufstehen“ von $50 \%$ auf $71 \%$, bei „Die Behandlung beeinflusst mich in meiner Kleiderwahl“ von 33\% auf $58 \%$, bei „Ich muss meine Kleidung aufgrund der Behandlung öfter als normal wechseln oder waschen“ von $54 \%$ auf $71 \%$, bei „Im Rahmen meiner Behandlung habe ich oft Termine bei meinem Hautarzt“ von $42 \%$ auf $58 \%$, bei „Der Zeitaufwand für die Behandlung beeinträchtigt mein Leben“ von $50 \%$ auf $71 \%$ und bei „Durch die Behandlung fühle ich mich in der Pflege sozialer Kontakte stark eingeschränkt" von $71 \%$ auf $83 \%$. Zu Visite 1 waren zudem nur 33\% der Patienten durch die aktuelle Behandlung weniger oder eher weniger auf Arzttermine angewiesen, zu Visite 2 waren dies bereits 58\%. Der Anteil der Patienten, die für die Durchführung ihrer Schuppenflechte-Behandlung fremde Hilfe benötigten, änderte sich dagegen kaum: 79\% schätzten diese Aussage zu Visite 1 und $83 \%$ zu Visite 2 als unzutreffend oder eher unzutreffend ein.

Der DLQI-Gesamtwert lag zu Visite 1 durchschnittlich bei 11,2 (Median 9,0) und zu Visite 2 bei 4,4 (Median 3,0) ( Tab. 2).

Einen sehr großen oder extrem großen Einfluss der Hauterkrankung auf ihr Leben (DLQI-Gesamtwert $\geq 11$ ) verspürten dabei zu Visite $146 \%$, zu Visite 2 hingegen nur 8\% der Probanden. Folglich stieg auch der Anteil an Probanden, die gar keinen oder nur einen geringen Einfluss der Hauterkrankung auf ihr Leben (DLQI-Gesamtwert $\leq 5$ ) verspürten, zwischen Visite 1 und 2 von $29 \%$ auf $58 \%$.

Der durchschnittliche PGA sank zwischen Visite 1 und 2 von 3,2 (moderate) auf 1,75 (mild). Zu Visite 1 hatten $21 \%$ der Probanden eine schwere Schuppenflechte $(\mathrm{PGA}=4)$; zu Visite 2 hatte kein Proband eine schwere Psoriasis mehr; dahingegen stieg der Anteil der Probanden, die höchstens eine milde Ausprägung der Schuppenflechte zeigten (PGA $\leq 2)$, zwischen Visite 1 und 2 von $0 \%$ auf $76 \%$ ( Abb. 4). 


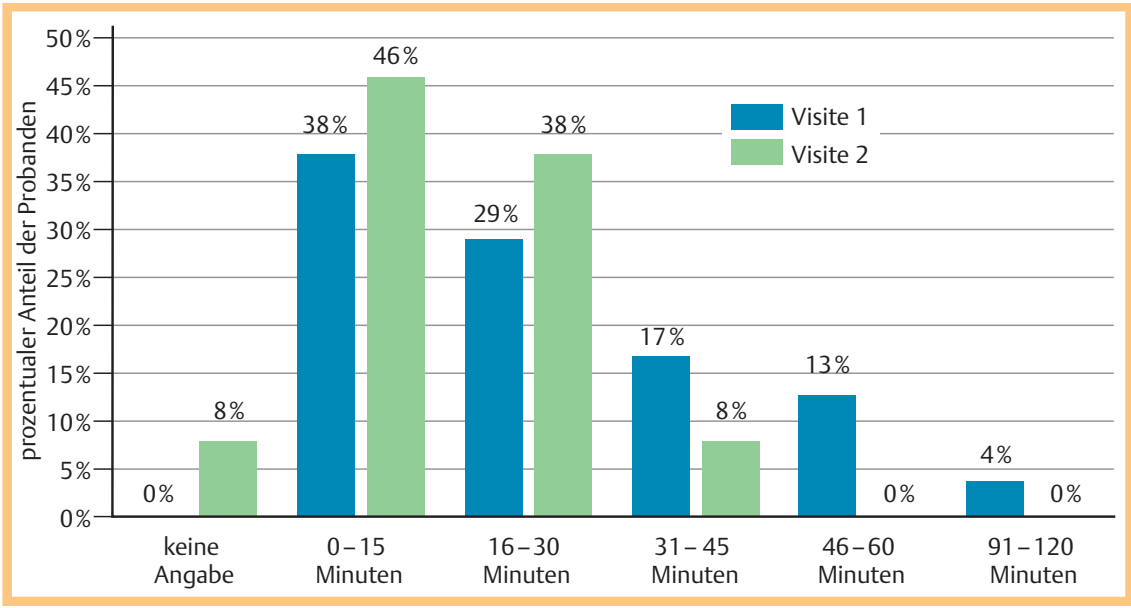

Abb. 3 Behandlungszeitaufwand pro Tag.

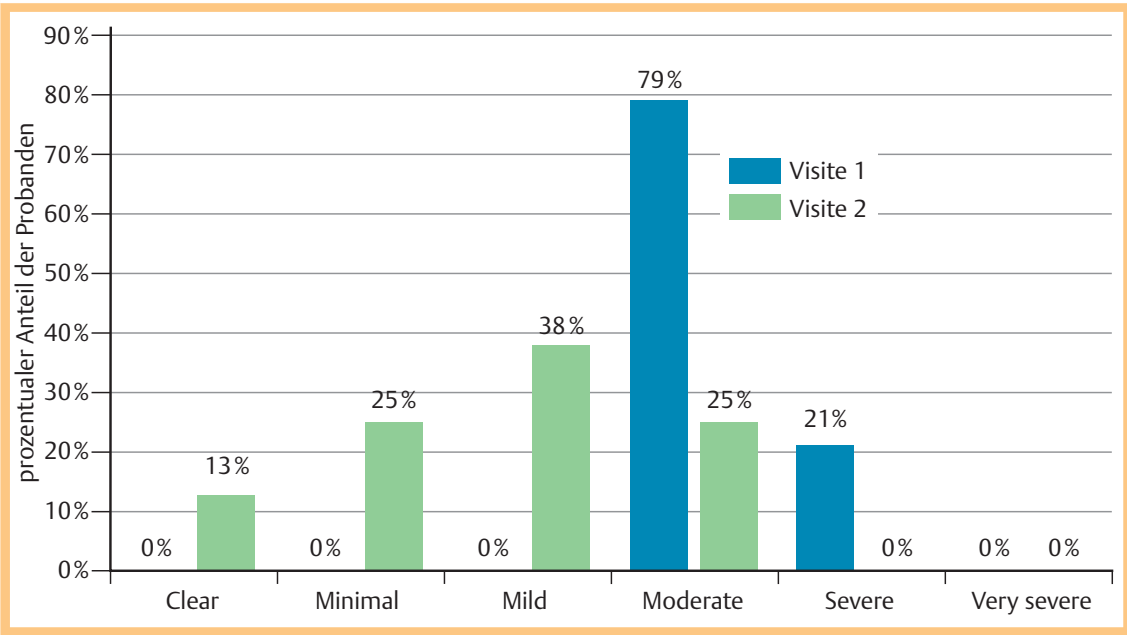

Abb. 4 Physician's Global Assessment.

Tab. 2 DLQI Total Score.

\begin{tabular}{|c|c|c|}
\hline & Visite 1 & Visite 2 \\
\hline$n$ & 24 & 22 \\
\hline Mean & 11.2 & 4.4 \\
\hline SD & 7.36 & 4.28 \\
\hline Median & 9.0 & 3.0 \\
\hline Min & 1 & 0 \\
\hline Max & 24 & 14 \\
\hline
\end{tabular}

Insgesamt wurden elf unerwünschte Ereignisse, von denen drei als schwer, die übrigen als moderat eingestuft wurden, bei insgesamt fünf Probanden im Verlauf der Studie dokumentiert $(3 \times$ Durchfall, $3 \times$ gastrointestinale Krämpfe, $2 \times$ Flush-Symptomatik, $1 \times$ Kreislaufkollaps, $1 \times$ Müdigkeit, $1 \times$ Gelenkbeschwerden). Zehn unerwünschte Ereignisse wurden als wahrscheinlich, eines als möglicherweise in Zusammenhang mit Einnahme der Studienmedikation stehend gewertet. Alle Probanden erholten sich vollständig von den unerwünschten Ereignissen, zwei beendeten die Studie deswegen jedoch vorzeitig.

Es trat kein schwerwiegendes unerwünschtes Ereignis auf.

\section{Diskussion}

Die Ergebnisse dieser nicht-interventionellen Studie (NIS) weisen darauf hin, dass eine Therapie mit Fumaderm ${ }^{\circledR}$ mit begleitender optionaler Lokaltherapie weniger zeitaufwendig ist als eine topische und/oder Fototherapie. Nicht nur nahm die Zeit, die ein Patient täglich für die Schuppenflechte-Behandlung aufwenden musste, nach Therapieumstellung auf Fumaderm ${ }^{\circledR}$ durchschnittlich ab, auch die Bewertung von sieben der acht Aussagen zur Belastung durch die Psoriasis-Behandlung unter besonderer Berücksichtigung des Zeitaufwandes deutete auf einen Rückgang der zeitlichen Belastung des Probanden hin.

Parallel dazu konnte eine deutliche Verbesserung der Lebensqualität und der Schwere der Schuppenflechte-Erkrankung durch Erhebung des DLQI und des PGA nach nur 12-wöchiger Behandlung mit Fumaderm ${ }^{\circledR}$ festgestellt werden.

Bei der im Rahmen dieser 12-wöchigen Studie bereits beobachteten Besserung der Hautveränderungen nach Therapieumstellung auf Fumaderm ${ }^{\circledR}$ und optionaler topischer Behandlung muss zudem berücksichtigt werden, dass sich die maximale Wirkung von Fumaderm ${ }^{\circledR}$ gemäß vorhergehenden Studien erst nach 24 wöchiger Behandlung einstellt und somit eine weitere Verbesserung des Hautbefundes bei den behandelten Patienten zu erwarten ist [7].

Bei den im Verlauf der Studie dokumentierten unerwünschten Ereignissen handelte es sich größtenteils um bekannte und zu erwartende Nebenwirkungen von Fumaderm ${ }^{\circledR}$. Ihre Häufigkeit lag 
dabei weitestgehend innerhalb des in der deutschen Fachinformation von Fumaderm ${ }^{\circledR}$ initial/Fumaderm ${ }^{\circledR}$ beschriebenen Rahmens [8]. Die Therapieabbruchrate aufgrund unerwünschter Ereignisse war mit zirka $8 \%$ eher niedriger als in vorausgehenden Studien, in denen bis zu einem Drittel der Patienten die Behandlung mit Fumaderm ${ }^{\circledR}$ vorzeitig beendete $[9,10]$.

Zusammenfassend lässt sich also auch in dieser Studie die von Blome et al. [6] beschriebene Korrelation zwischen sinkendem Behandlungszeitaufwand und steigender Lebensqualität bei Patienten mit Schuppenflechte nachweisen. Parallel dazu konnte ein deutlicher Rückgang des PGAs gleichbedeutend mit einem hohen Wirkungsgrad von Fumaderm ${ }^{\circledR}$ nach nur 12-wöchiger Behandlung beobachtet werden. In welchem Ausmaß der Rückgang des Zeitaufwandes bzw. die Besserung der Hautveränderungen zur Steigerung der Lebensqualität dabei jeweils beigetragen haben, lässt sich durch das Design dieser Studie nicht beurteilen. Diese und vorhergehende Studien legen jedoch nahe, dass neben der reinen Effizienz eines Medikamentes auch immer seine Praktikabilität - vor allem im Bezug auf den Zeitaufwand - berücksichtigen werden sollte, um für den Psoriasis-Patienten eine größtmögliche Verbesserung der Lebensqualität zu erreichen.

\section{Interessenkonflikt}

\section{$\nabla$}

Die Studie wurde von Prof. Dr. med. Kristian Reich als Sponsor initiiert und am Dermatologikum Hamburg, Drehbahn 1-3, 20354 Hamburg, durchgeführt. Datenauswertung und Erstellung des Abschlussberichtes erfolgte durch die SCIderm GmbH, Esplanade 6, 20354 Hamburg (Geschäftsführerin: Dr. phil. Ina Zschocke). Das Projekt wurde finanziell von der Firma Biogen Idec Inc. gesponsert.

Herr Prof. Dr. med. Kristian Reich hat Honorare als Berater und als Gastredner von Biogen Idec Inc. erhalten und an Studien teilgenommen, die von Biogen Idec Inc. gesponsert wurden.

Herr Dr. med. Marcus Neureither arbeitet als Leiter der Medical Affairs Abteilung Dermatologie bei der Firma Biogen Idec Inc. Frau Dr. med. Christina Kahl arbeitet als Fachärztin für Dermatologie und Venerologie am Dermatologikum Hamburg und als Prüfärztin bei der SCIderm GmbH. Darüber hinaus bestehen keine weiteren Interessenkonflikte.

\section{Abstract}

Time Management and Quality of Life After Change of Therapy from Topical Treatment and/or Phototherapy to Fumaderm ${ }^{\circledR}$ in Patients with Psoriasis

$\nabla$

Introduction: Psoriasis often is accompanied by an impairment of the patient's quality of life. The reduction in quality of life correlates most strongly with the time the patients have to spend to treat their skin disease. Therefore, by means of this study it should be investigated whether a switch from topical and/or phototherapy to a systemic treatment with Fumaderm ${ }^{\circledR}$ with optional topical therapy leads to a reduction of the treatment burden - in special consideration of the time effort - and to an improvement of the patient's quality of life and the severity of the skin lesions.

Material and Method: Within this non-interventional study, patients with a moderate to very severe psoriasis undergoing a switch from topical and/or phototherapy to a systemic treatment with Fumaderm ${ }^{\circledR}$ with optional topical therapy were observed. Before and 12 weeks after treatment with Fumaderm ${ }^{\circledR}$, PGA, DLQI and the time the patients had to invest per day to treat their psoriasis were assessed. Furthermore, it was investigated to which extent eight statements concerning the treatment burden - under special consideration of the time effort - applied for the respective patients.

Results: After 12 weeks of treatment with Fumaderm ${ }^{\circledR}$, a reduction of the time, the 24 included patients had to invest on the average to treat their skin disease per day, could be found. Also, comparative analyses of seven of the eight statements concerning the treatment burden indicated an improvement of the time burden as a consequence of the treatment switch. In the course of the study, the PGA score decreased on the average from 3.2 to 1.75 and the DLQI total score from 11.2 to 4.4 . Altogether, 11 adverse events, all of them at least possibly related to the administration of Fumaderm ${ }^{\circledR}$, were documented.

Discussion: The results of this study portend, that a therapy with Fumaderm $^{\circledR}$ and optional topical therapy is less time consuming than a topical and/or phototherapy. Furthermore, a clear improvement of the patient's quality of life and of the psoriasis severity could be shown after 12 weeks of treatment with Fuma$\operatorname{derm}^{\circledR}$.

\section{Literatur}

1 Schaefer I, Rustenbach SJ, Zimmer L, Augustin M. Prevalence of skin diseases in a cohort of 48,665 employees in Germany. Dermatology 2008; 217 (2): $169-172$

2 Rapp SR, Feldman SR, Exum ML et al. Psoriasis causes as much disability as other major medical diseases. J Am Acad Dermatol 1999; 41: 401 407

3 Torres RA, Silva SA, Magalhatildees RF et al. Comparison of quality of life questionnaires and their correlation with the clinical course of patients with psoriasis. An Bras Dermatol. 2011; 86 (1): 45 - 49

4 Hong J, Koo B, Koo J. The psychosocial and occupational impact of chronic skin disease. Dermatol Ther 2008; 21 (1): 54 - 59

5 Finlay AY, Khan GK. Dermatology Life Quality Index (DLQI) - a simple practical measure for routine clinical use. Clin Exp Dermatol 1994; 19 (3): $210-216$

6 Blome C, Simianer S, Purwins $S$ et al. Time needed for treatment is the major predictor of quality of life in psoriasis. Dermatology 2010; 221 (2): $154-159$

7 Reich K, Thaci D, Mrowietz $U$ et al. Efficacy and safety of fumaric acid esters in the long-term treatment of psoriasis - a retrospective study (FUTURE). J Dtsch Dermatol Ges 2009; 7: 603-611

8 Deutsche Fachinformation: Fumaderm ${ }^{\circledR}$ initial/Fumaderm ${ }^{\circledR}$ von Biogen Idec GmbH, Stand: Februar 2009

9 Harries MJ, Chalmers RJ, Griffiths CE. Fumaric acid esters for severe psoriasis: a retrospective review of 58 cases. Br J Dermatol 2005; 153 (3): 549-551

10 Wain EM, Darling MI, Pleass RD, Barker JN, Smith CH. Treatment of severe, recalcitrant, chronic plaque psoriasis with fumaric acid esters: a prospective study. Br J Dermatol 2010; 162 (2): 427-434 